\title{
Guidelines for genetic skeletal dysplasias for pediatricians
}

\author{
Sung Yoon Cho, MD, PhD, \\ Dong-Kyu Jin, MD, PhD
}

Department of Pediatrics, Samsung Medical Center, Sungkyunkwan University School of Medicine, Seoul, Korea

\begin{abstract}
Skeletal dysplasia (SD) is a kind of heterogeneous genetic disorder characterized by abnormal growth, development, differentiation, and maintenance of the bone and cartilage. The patients with SD most likely to be seen by a pediatrician or orthopedic surgeon are those who present with short stature in childhood. Because each category has so many diseases, classification is important to understand SD better. In order to diagnose a SD accurately, clinical and radiographic findings should be evaluated in detail. In addition, genetic diagnosis of SD is important because there are so various SDs with complex phenotypes. To reach an exact diagnosis of SDs, cooperative approach by a clinician, a radiologist and a geneticist is important. This review aims to provide an outline of the diagnostic approach for children with disproportional short stature.
\end{abstract}

Keywords: Short stature, Skeletal dysplasia, Exome sequencing
Received: 21 December, 2015 Accepted: 21 December, 2015

Address for correspondence: Dong-Kyu Jin, MD, PhD

Department of Pediatrics, Samsung Medical Center, Sungkyunkwan University School of Medicine, 81 Irwon-ro, Gangnam-gu, Seoul 06351, Korea

Tel: +82-2-3410-3525

Fax: +82-2-3410-0043

E-mail: jindk@skku.edu

\section{What is a skeletal dysplasia?}

Skeletal dysplasias (SDs) are a large, heterogeneous group of genetic disorders characterized by abnormal growth, development, differentiation, and maintenance of the bone and cartilage. Albeit individually rare, collectively the birth incidence is estimated to be about 1:5,000 live births, which represents $5 \%$ of children born with a birth defect ${ }^{1)}$. The true incidence is likely to be higher due to under-diagnosis ${ }^{2)}$. SD typically presents with short stature in childhood; however due to its heterogeneity, musculoskeletal effects range in severity from premature arthritis in average height individuals to severe short stature with death in the perinatal period ${ }^{3)}$. The patients most likely to be seen by a pediatrician or orthopedic surgeon are those who present with short stature in childhood. The spine and limbs are frequently affected, in addition to musculoskeletal abnormalities in hearing, vision, and neurological, respiratory, cardiac, and renal function, as well as psychological problems ${ }^{4-8)}$. It is sometimes unclear whether the major cause of growth failure is skeletal or systemic. Endocrine, cardiac, respiratory, and renal abnormalities should be ruled out. However, patients with these abnormalities usually show proportionately short stature whereas patients with SDs have disproportionate short stature. Moreover, some genetic syndromes cause prenatal growth failure but should be easily distinguishable on the basis of associated features, such as dysmorphic facies, developmental delay, and, if necessary, features seen on radiographs ${ }^{9}$.

\section{Why is the classification of SD important?}

Because each category has so many diseases, classification is important to understand SD better. In the 1960s, patients with disproportional short stature were suspected as either achondroplasia (short-limbed type of SD) or Morquio syndrome (short-trunked type of $\mathrm{SD})^{10)}$. As numerous entities have been delineated not to fit these two SDs, experts created a systematic approach. In the 1970s, the categories were merely clinical and descriptive. As the 
genetic background and the pathogenesis of various entities were identified, the categories evolved into a combination of clinical, radiological, and genetic information. In the latest 2015 version of the nosology ${ }^{11}$, the overall number of disorders has decreased from 456 to 436 but the number of groups has increased from 40 to 42 and the number of genes from 226 to 364 compared to those of the 2011 version. The classification has provided a lot of help in basic research as well as in the identification of disease-responsible genes.

In the clinical field, however, physicians seeing patients with short stature face the complex list of many diseases. Classification of SDs is continually evolving and it is not always possible to classify patients into a defined group. Therefore, exact evaluation of clinical and radiographic findings is important to reach an accurate diagnosis of a SD. This review aims to provide an outline of the diagnostic approach for children with disproportionate short stature.

\section{How can we reach a diagnosis of SD?}

\section{Clinical evaluation}

1) History taking when we meet any patients with disproportionate short stature

Prior to physical examination, accurate history about the onset time of short stature is essential. When a child with disproportionate short stature presents, a focused history taking is important to catchany clue to the differential diagnosis. This includes prenatal history and birth length. For example, patients with achondroplasia usually present with remarkable short stature at birth ${ }^{12)}$ whereas those with pseudoachondroplasia have a normal birth length with subsequent growth failure within 2 years old ${ }^{13)}$. Recently, SDs, even the nonlethal diseases, can be detected on prenatal ultrasound and it is worth to ask whether any discrepancy was observed between fetal size and gestational dates if the ultrasound scans were carried out ${ }^{14)}$. The family history and pedigree are also important. If the family has

\begin{tabular}{l} 
Table 1. History taking for skeletal dysplasia \\
\hline When the short stature was first recognised \\
Prenatally or later during childhood \\
Antenatal history \\
Femora or humeri length $<5$ th centile or -2 standard deviation score \\
in the 2 nd trimester \\
Family history and pedigree \\
Parental height, other family members affected, parental consanguinity \\
Body measures at birth \\
Length, weight, and head circumference \\
Joint pain \\
Ligamentous laxity or joint contracture \\
Susceptibility to infections \\
Age at starting to walk independently \\
Fracture or orthopedic surgery history \\
Growth velocity
\end{tabular}

other patients with SD besides the proband, this will be helpful to assess the inheritance mode of the disease. Parental heights are useful if the child is considered as having familial short stature. History taking for SD is summarized in Table 1.

\section{2) Physical examination}

A careful examination by a clinical expert can narrow down the possible diseases even before the radiologic evaluation. On physical examination, growth parameters such as height, weight, and head circumference are essential. In addition, sitting height, upper/lower segment ratio, and arm span are sometimes important when a child with short stature should be evaluated. The lower segment can be measured from symphysis pubis towards the sole medially to the heel. The upper segment can be calculated by subtracting the lower segment from the total height. The mentioned ratios change with age. A patient with a short trunk has a decreased upper/lower segment ratio, while a short statured patient with normal trunk and relatively short limbs may have an increased upper/lower segment ratio ${ }^{15}$. Disproportionate short stature is sub-divided into short-trunk or short-limb varieties. In the short-limb type, rhizomelia refers to proximal segment shortening (humerus and femur), mesomelia refers to middle segment shortening (radius, ulna, tibia, and fibula) and acromelia to distal segment shortening (hand and foot). Micromelia refers to abnormally short limb(s) and brachydactyly is short digits. These descriptions help in differential diagnosis.

A general physical examination is also importmant because ancillary signs such as facial dysmorphism can give us any diagnostic clue. Macrocephaly, frontal bossing, midface hypoplasia, and short upturned noses are characteristics of achondroplasia ${ }^{12}$; cleft palate and micrognathia are characteristics of type II collagenopathies; midface hypoplasia with flat nasal bridge and gray iris color are characteristics of

\section{Table 2. Physical examination for skeletal dysplasia}

Anthropometric measurements
Height, weight, head circumference (standard deviation score),
upper/lower segment ratio, sitting height, and arm span
Hyperextensibility
Facial dysmorphism
Blue sclera
Dentinogenesis imperfecta
Pectus excavatum
Lordosis, scoliosis
Genu varus/valgum
Brachydactyly
Acro/meso/resomelic
Hepatosplenomegaly
Walking pattern
Limitation of movement
Nail, hair, skin
Hearing
Vision
Cognitive impairment


acrodysostosis $^{16)}$; and dentinogenesis imperfecta is a characteristics of odontochondrodysplasia ${ }^{17)}$. Laboratory data and other clinical assessments such as hair, nail, cleft palate, vision, hearing, and even abnormalities of the internal organs (kidneys, liver, and spleen) are important in SD evaluation. Physical examination for SD is summarized in Table 2.

Children with disproportional short stature may to be affected by a kind of SD. However, the abnormal body proportions can be missed at the first exam. It is therefore important to measure body proportions exactly. Some SDs with abnormalities of bone mineralization, such as osteogenesis imperfecta, osteopetrosis, and hypophosphatasia, may show near normal body proportions.

\section{Radiological assessment}

After obtaining family history, making a pedigree in detail, and performing a physical examination, radiological assessment is likely to reach a diagnosis of the patient in most SDs because almost disease have distinctive radiological features in growing bones and cartilages. Therefore, a complete skeletal survey should be performed (Table 3 ). The radiographic patterns may include any or all of the following: spondylo, epiphyseal, metaphyseal, and diaphyseal dysplasia. These patterns helps to narrow down the possible group of dysplasias ${ }^{18)}$. Epiphyseal dysplasia is characterized by absent, small, or irregularly ossified epiphyses. Metaphyseal dysplasia is characterized by irregular, widened, or flared metaphyses. Diaphyseal dysplasia is characterized by diaphyseal widening, sclerosis, cortical thickening, or medullary narrowing or expansion. Besides the pattern of skeletal abnormalities, the affected region can be useful to narrow the differential diagnosis. Associated spinal involvement denotes a spondylo-epiphysealdysplasia, spondylo-metaphyseal-dysplasia, or spondyloepi-meta-physeal-dysplasia. Radiographs should also be analyzed for bone age maturation (e.g., Laron syndrome) and abnormal mineralization (osteogenesis imperfecta and hypophosphatasia) ${ }^{19)}$.

In children with proportionate short stature, the differential diagnosis includes familial short stature, constitutional growth delay, a kind of endocrinopathies including growth hormone

Table 3. Radiographic evaluation in children with disproportional short stature

\begin{tabular}{lc}
\hline Region & View \\
\hline Skull & PA, lateral \\
T-L spine & AP, lateral \\
Thorax & AP \\
Pelvis & AP \\
Upper limb & AP \\
Both hands and wrists & PA \\
Lower limb & AP \\
Foot & AP, lateral \\
\hline
\end{tabular}

PA, posteroanterior view; AP, anterioposterior view; lateral, lateral view; T-L, thoracolumbar.
(GH) deficiency, and a kind of dysmorphic syndromes. In these cases, the initial radiologic assessment should include a left hand and wrist radiograph for bone age determination and a forearm radiograph for haploinsufficiency of SHOX.

In the case of adults, prepubertal skeletal radiographs should be tried to obtain because diagnosis may be very difficult if the epiphyses have already fused to the metaphyses. Above all, radiological assessment requires professional experience in the field of pediatric radiology.

\section{Genetic diagnosis of SD}

Manydisease-responsible gene has discovered in these days thanks to the development of next-generation sequencing, particularly, whole exome sequencing (WES). This has led to both expansion and consolidation of the nosology. Genetic diagnosis of SDs is strongly needed because there are so many diseases with complex phenotypes and many individual variations even in the same disease $\mathrm{e}^{20)}$. Identification of the responsible gene and the pathogenesis of SDs are important to understand the diseases themselves and to improve diagnosis and treatment options.

Recently, many researchers have used WES and wholegenome sequencing (WGS) to identify new genes responsible for SD. However, these kinds of techniques are too expensive, and the lot of variants may be detected. Therefore, it is not easy to applicate those on general genetic testing. In this regard, screening of a certain number of genes by targeted exome sequencing (TES) could be a useful alternative. TES has a superior accuracy with high depth and is more simple to analyze and requires relatively low costs compared with WES and $\mathrm{WGS}^{21,22)}$. SD is a good candidate disease for TES, because many SDs are caused by various pathogenic variants scattered along the many or huge genes of matrix proteins without hotspots, and it is often unclear which gene should be tested first.

The most appropriate genetic test depends on the patients' clinical impression. When a patient is suspected as having a SD with only a single known pathogenic variant(s) (e.g., G380R in FGFR3 of achondroplasia or R836C in COL1A1 of infantile cortical hyperostosis), Sanger sequencing for the specific region would be the optimal test to confirm the molecular

Table 4. Candidate skeletal dysplasia for targeted exome sequencing

\begin{tabular}{lc}
\hline Disease & \multicolumn{1}{c}{ Gene } \\
\hline Osteogenesis imperfecta & COL1A1, COL1A2, IFITM5, CRTAP, P3H1, \\
& FKBP10, LEPRE1, PLOD2, PPIB, SERPINF1, \\
& SERPINH1, SP7, TMEM38B, WNT1 \\
Multiple epiphyseal dysplasia COMP, MATN3, COL9A1, COL9A2, COL9A3, & DTDST \\
& CLCN7, TNFSF11, TCIRG1, OSTM1, \\
Osteopetrosis & TNFRSF11A, PLEKHM1, CA2, LRP5, IKBKG, \\
& FERMT3, RASGRP2 \\
Ehlers-Danlos syndrome & COL5A1, COL5A2, COL3A1, PLOD1, \\
& COL1A1, COL1A2, ATP7A, SLC39A13 \\
Type II collagenopathy & COL1A2 \\
\hline
\end{tabular}


diagnosis. When a patient is suspected as having a SD with multiple candidate variants in a single or multiple candidate causative gene(s), TES has merit over Sanger sequencing because all candidate genes can be screened simultaneously. The candidate diseases for TES are shown in Table 4. When a patient is suspected as having a SD with mutations dispersed widely in huge genes, such as COL2A1 (67 exons) of type II collagenopathy, TES has advantages over Sanger sequencing because of its cost-effectiveness. Any candidate variant should be validated by Sanger sequencing and should pass segregation study. If the candidate variant is previously reported, the variant can be confirmed as the responsible variant. If the candidate variant is novel, the variant is highly likely to be responsible, but the pathogenicity of the novel variant should be validated by either some prediction program, frequency in the database, or in vitro study if needed. If any uncovered region is included in a certain candidate gene, it should be checked by Sanger sequencing.

If no candidate variant was found by TES, there may be several reasons: no genetic disorder, not discovered causative gene yet, variants in deep intro or regulatory/enhancer sequences, large insertions or deletions, and chromosomal anomalies $^{22)}$. In these cases, the next step requires WES, WGS, array comparative genomic hybridization, multiplex ligationdependent probe amplification, or fluorescence in situ hybridization.

\section{Management of SD}

SD requires multidisciplinary management by a number of specialists to address specific complications associated with each problem. Treatment principles for orthopedic complications are to prevent or correct limb deformity, to stabilize joint laxity, to prevent fracture, to equalize limb lengths, to replace joints

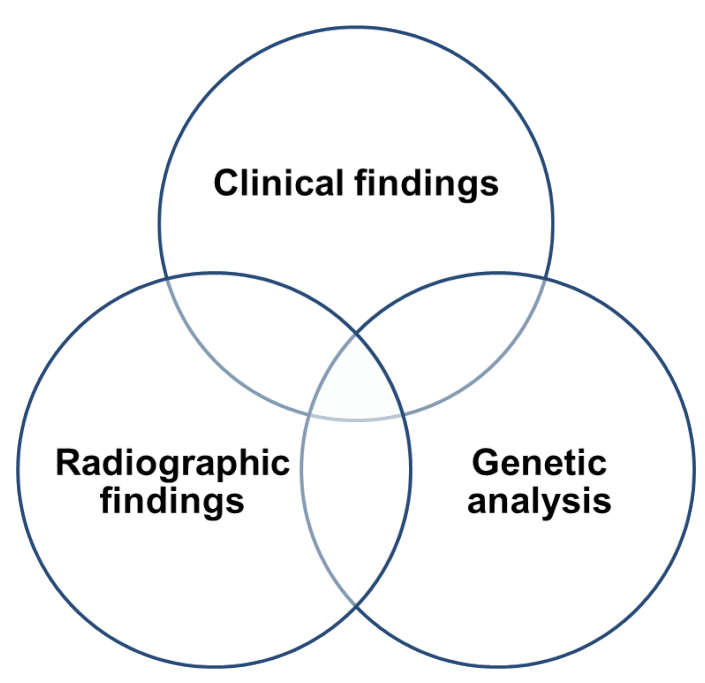

Fig. 1. Cooperative approach by a clinician, a radiologist and a geneticist is important for diagnosis of skeletal dysplasia. for arthritis, and to decompress, realign, and stabilize the spine to prevent deformity and neurological injury. For those with a small chest circumference, respiratory support is essential. Children should be screened regularly for hearing and visual impairment. Pain should be specifically asked about because it can reduce quality of life. Medical treatment should be used to minimize complications, such as fractures, or to increase the final height through use of GHs. The effectiveness of GH treatment in patients with SD is controversial and dependent on the type of SD. There have been some reports of positive results in achondroplasia and hypochondroplasia; however, GH treatment was not effective in other type of SD, such as pseudoachondroplasia and spondyloepiphyseal dysplasia. Moreover, GH treatment can worsen body disproportion, necessitating surgical limb lengthening. The treatment for osteogenesis imperfecta is bisphosphonate with calcium and vitamin $\mathrm{D}$ supplementation if the child is deficient to increase bone mineral density and reduce the fracture risk.

\section{Conclusions}

$\mathrm{SD}$ is a large, heterogeneous group of genetic disorders that typically present with short stature in childhood. Accurate diagnosis based upon clinical and radiographic features is important to predict final height, expected complications, and treatment, and to allow specific genetic counselling. Genetic diagnosis of SDs is important because there are so many diseases with complex phenotypes. In order to reach an exact diagnosis of SDs, cooperative approach by a clinician, a radiologist and a geneticist is important (Fig. 1). A multidisciplinary approach is required for patients with SDs.

\section{Conflict of interest}

No potential conflict of interest relevant to this article was reported.

\section{References}

1. Orioli IM, Castilla EE, Barbosa-Neto JG. The birth prevalence rates for the skeletal dysplasias. J Med Genet 1986;23:328-32.

2. Unger S. A genetic approach to the diagnosis of skeletal dysplasia. Clin Orthop Relat Res 2002:32-8.

3. International nomenclature and classification of the osteochondrodysplasias (1997). International Working Group on Constitutional Diseases of Bone. Am J Med Genet 1998;79:376-82.

4. Bober MB, Taylor M, Heinle R, Mackenzie W. Achondroplasia-hypochondroplasia complex and abnormal pulmonary anatomy. Am J Med Genet A 2012;158A:233641.

5. Digilio MC, Marino B, Giannotti A, Dallapiccola B. Atrioventricular canal defect and postaxial polydactyly 
indicating phenotypic overlap of Ellis-van Creveld and Kaufman-McKusick syndromes. Pediatr Cardiol 1997;18:74-5.

6. Khan S, Hinks J, Shorto J, Schwarz MJ, Sewell WA. Some cases of common variable immunodeficiency may be due to a mutation in the SBDS gene of Shwachman-Diamond syndrome. Clin Exp Immunol 2008;151:448-54.

7. Kiel EA, Frias JL, Victorica BE. Cardiovascular manifestations in the Larsen syndrome. Pediatrics 1983;71:942-6.

8. Makitie O, Pukkala E, Kaitila I. Increased mortality in cartilage-hair hypoplasia. Arch Dis Child 2001;84:65-7.

9. KL J. Smith's Recognizable Patterns of Human Malformation. 5th ed. Philadelphia: WB Saunders, 1997.

10. Krakow D, Rimoin DL. The skeletal dysplasias. Genet Med 2010;12:327-41.

11. Bonafe L, Cormier-Daire V, Hall C, Lachman R, Mortier G, Mundlos S, et al. Nosology and classification of genetic skeletal disorders: 2015 revision. Am J Med Genet A 2015 Sep 23 [Epub]. http://dx.doi.org/10.1002/ajmg.a.37365.

12. Hunter AG, Bankier A, Rogers JG, Sillence D, Scott CI Jr. Medical complications of achondroplasia: a multicentre patient review. J Med Genet 1998;35:705-12.

13. McKeand J, Rotta J, Hecht JT. Natural history study of pseudoachondroplasia. Am J Med Genet 1996;63:406-10.

14. Garjian KV, Pretorius DH, Budorick NE, Cantrell CJ, Johnson DD, Nelson TR. Fetal skeletal dysplasia: threedimensional US: initial experience. Radiology 2000; 214:717-23.
15. Mortier GR. The diagnosis of skeletal dysplasias: a multidisciplinary approach. Eur J Radiol 2001;40:161-7.

16. Kaname T, Ki CS, Niikawa N, Baillie GS, Day JP, Yamamura $\mathrm{K}$, et al. Heterozygous mutations in cyclic AMP phosphodiesterase-4D (PDE4D) and protein kinase A (PKA) provide new insights into the molecular pathology of acrodysostosis. Cell Signal 2014;26:2446-59.

17. Unger S, Antoniazzi F, Brugnara M, Alanay Y, Caglayan A, Lachlan $\mathrm{K}$, et al. Clinical and radiographic delineation of odontochondrodysplasia. Am J Med Genet A 2008;146A: 770-8.

18. DL R, RS L. Principles and practice of medical genetics. 3 ed. New York: Churchill Livingstone, 1997.

19. Sewell MD, Chahal A, Al-Hadithy N, Blunn GW, Molloy S, Hashemi-Nejad A. Genetic skeletal dysplasias: a guide to diagnosis and management. J Back Musculoskelet Rehabil 2015;28:575-90.

20. Ikegawa S. Genetic analysis of skeletal dysplasia: recent advances and perspectives in the post-genome-sequence era. J Hum Genet 2006;51:581-6.

21. Park JH, Kim NK, Kim AR, Rhee J, Oh SH, Koo JW, et al. Exploration of molecular genetic etiology for Korean cochlear implantees with severe to profound hearing loss and its implication. Orphanet J Rare Dis 2014;9:167.

22. Bae JS, Kim NK, Lee C, Kim SC, Lee HR, Song HR, et al. Comprehensive genetic exploration of skeletal dysplasia using targeted exome sequencing. Genet Med 2015 Sep 24 [Epub].http://dx.doi.org/10.1038/gim.2015.129. 